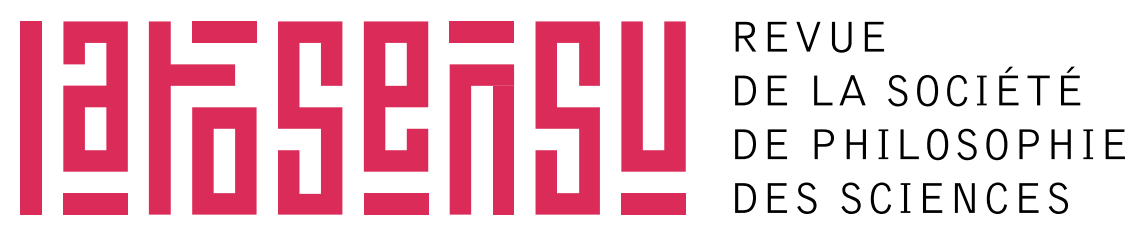

Vol $3 N^{\circ} 12016$

DOI http://dx.doi.org/10.20416/lsrsps.v3i1.6o3

Carl Hoefer

OBJective

CHANCE:

NOT PROPENSITY,

MAYBE

DETERMINISM

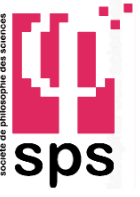

SOCIÉTÉ DE PHILOSOPHIE DES SCIENCES (SPS) 


\section{Carl Hoefer}

\section{OBJECTIVE CHANCE: NOT PRO- PENSITY, MAYBE DETERMINISM}

\author{
Sommaire \\ 1 - Introduction \\ 2 - The puzzle, in more depth \\ 3 - The dialectics of primitive \\ chance \\ 4 - Chances from Determin- \\ ism: the Solution? \\ 5 - Conclusion
}

One currently popular view about the nature of objective probabilities, or objective chances, is that they - or some of them, at least - are primitive features of the physical world, not reducible to anything else nor explicable in terms of frequencies, degrees of belief, or anything else. In this paper I explore the question of what the semantic content of primitive chance claims could be. Every attempt I look at to supply such content either comes up empty-handed, or begs important questions against the skeptic who doubts the meaningfulness of primitive chance claims. In the second half of the paper I show that, by contrast, there are clear, and clearly contentful, ways to understand objective chance claims if we ground them on deterministic physical underpinnings.

Key words: chance, probability, propensity, primitive chance, subjective probability, determi-

nism, indeterminism, Humeanism, frequentism

\section{1 - Introduction}

Ever since I first encountered the notion of objective probability or objective chance, taken as a property of certain systems in the world, I have been troubled by a deep puzzlement about how to understand this idea. Consider a system or setup $S$ to which we attribute an objective chance $x$ of yielding outcome $o_{1}$ and $y$ of yielding outcome $o_{2}$. In its crudest form, my puzzlement expressed itself in questions of the following sort: "How does system $S$ decide when to produce outcome o1 and when to produce outcome $\mathrm{O}_{2}$ ?" and "How does S keep track of the results of previous $S$-chancy events, to make sure the frequencies of $o_{1}$ and $o_{2}$ build up in the right way?" I immediately imagined a hidden gremlin in $S$ that flips a coin (biased to the right degree, to match $x$ and $y$ ) to determine which way to go. But that clearly just postpones the puzzlement momentarily; it comes right back concerning the gremlin's coin flips; and anyway, gremlins and other homunculi are not respectable explanatory tools, in science or in philosophy.

Despite the phrasing of the two questions just above, my puzzlement is not based on a misguided anthropomorphizing of chance systems, attributing to them beliefs and desires. The puzzle is about how to understand a bare posit - basically, just assigning a set of numbers to a chance setup's outcomes - as somehow able to explicate, to justify and make reasonable, all the riches of derived mathematical consequences and practical applications that probability theory affords us.

Despite years of thinking about probability and chance, I've never been able to shake the feeling that I don't understand what is meant by those who postulate bare, unanalyzable and underived primitive objective chances, or chancy fundamental laws. In this paper my aim is to articulate my puzzlement as clearly as I can, and hopefully induce some readers to share it. And by exploring the dialectics of the debates between primitive chance advocates and skeptics, I will try to show that alternative understandings of chance, that reduce objective chance facts to facts of certain other kinds, are very much to be preferred, if they can be crafted in ways that don't face insuperable difficulties of their own. As we will see, it seems that this can be done in more than one way, especially if we assume underlying determinism. Hence the title of this paper.

\section{2 - The puzzle, in more depth}

There has been widespread agreement for the past 100 years or so that probability is a concept that, while well-enough defined mathematically by various systems of axioms (e.g. Kolmogorov's), is not automatically well understood when attributed to a situation or event in, or a proposition about, the real world. Instead, probability statements are in need of an interpretation. Alan Hájek's Stanford Encyclopedia of Philosophy (2012) article "Interpretations of Probability" canvasses the standard proposals that have been made for how to interpret all, or some parts, of our probability-talk, and the problems that each faces. Most prominently, probability-talk is often cashed out in terms of the frequencies of certain conditions or events obtaining, or in terms of the degrees of belief of agents in conditions of uncertainty. But since the work of Peirce at least in the early 19oos there have been proposals that certain types of probability-talk be taken as primitive and basic, not to be cashed out or interpreted in terms of anything else. The idea is that there may be single events and/or types of events that are simply, irreducibly chancy: indeterministic, random, and only probabilistically predictable, i.e., associable with specific numerical chances in between zero and one. Usually, when contrasted with other 


\section{OBJECTIVE CHANCE: NOT PROPENSITY, MAYBE DETERMINISM}

views about probability, this proposal is called the "propensity interpretation". But this is a misnomer since the view is not an interpretation (= translation into other terms) so much as a flat-footed insistence that no interpretation in other terms can be given. ${ }^{1}$

While some philosophers find the propensity ${ }^{2}$ view intuitive and relatively easy to understand, others accompany me in being puzzled about what could be meant by the postulation of primitive chances. Prominent among the puzzled are those who have (broadly) Humean leanings, such as van Fraassen or David Lewis. In a famous passage we will examine later, Lewis indicated his skepticism about primitive chances by labeling them 'whatnots'. But it may be that such skepticism is confined to a minority of contemporary philosophers; let's see whether that can be remedied.

The basic question I want to pose and explore is this: what is the meaning of a putative primitive chance statement? What is the content of a statement such as $\operatorname{Pr}_{S}(O)=.4$, (where $S$ is an irreducibly chancy setup or situation, and $O$ one of the possible outcomes), or of a putative chancy fundamental law statement such as $\operatorname{Pr}_{S}\left(G_{i}\right)=x_{i}$ (where the $G_{i}$ are the possible "outcomes" of the chance process covered by the law, and the $x_{i}$ their objective chances)? What sort of claim are these statements supposed to be making about the world - and in particular, what differentiates them from identical statements with numerically different $x$ 's on the right? How can we distinguish a possible world in which $\operatorname{Pr}_{S}(\mathrm{O})=0.4$ is true from one in which $\operatorname{Pr}_{S}(O)=0.55$ is true?

As we will see, it is not easy to answer these questions. In the next section we will explore the dialectics of attempts to give answers, and then consider the arguments of those who say no answer need be given.

\section{3 - The dialectics of primitive chance}

How and why should beliefs about objective chance help to shape our expectations of what will happen? This is the fundamental question about the concept of chance. I am going to argue that within the metaphysical point of view, the question cannot be answered at all. (van Fraassen 1989, p. 81)
The most powerful impetus for postulating primitive chances has come from the appearance in physics of an apparently fundamental and irreducible indeterminism in quantum theories. Outside of the realm of quantum-derived chances, the main type of system to which we are wont to ascribe definite, precise objective chances, but unwilling to consider them as mere frequencies, is classical gambling devices (coin flips, roulette wheels, card games, etc.). But as we will discuss later on, most gambling devices can probably be modeled as deterministic systems with reliably randomly-distributed initial conditions, and hence not genuine loci of primitive propensity-type chance. So let's begin the dialectic by trying to say what the content or meaning of a chancy-law statement could be.

On a traditional view of ordinary, non-probabilistic laws, one might say that the content of a law statement has two parts: an actuality-related part, and a modal part. The former part makes a claim about the way things are. Traditionally, we say that this part consists of a universal generalization something that can be made to look something like 'All F's are G's'. Newton's law of gravity can clearly be expressed in such a form, like so: "Whenever two solid bodies have masses $m_{1}$ and $m_{2}$ and their centers of gravity are separated by distance $r$, the force each body exerts on the other is $\frac{G m_{1} m_{2}}{r^{2}}$ and directed toward the first body's center of gravity." Together with other laws (notably $\boldsymbol{F}=m \boldsymbol{a}$ ) and bridge principles, this law describes how things evolve over time in the actual world, i.e., in what is often called the "Humean Mosaic". ${ }^{3}$ And the same goes for all other laws that are not probabilistic.

The modal part of laws is perhaps not so clearly or uncontroversially understood, but is nevertheless taken to be an important part of the content. A law of nature, we want to say, does not simply say that such-and-so does happen, but moreover says that - in some appropriate sense - such-and-so must happen, i.e., that it is in some sense necessary. Traditional accounts have never delivered a plausible and acceptable explication of this alleged necessity. Logical necessity seems too strong to most philosophers. "Metaphysical" necessity strikes most philosophers as too strong to be the right modality also, and it has the additional drawback of being a controversial notion in its own right. Jumping to the far end of the spectrum, most philosophers are also not content to dismiss or internalize the modality, making it a mere matter of our attitudes and habits - though a long tradition begin-

\footnotetext{
1 - In this paper I will indicate the target using phrases like "the propensity view", or "primitive chances" or "fundamental chancy laws", as appropriate given the context.

2 - The clearest example is to be found in Giere (1973); earlier papers by Popper are often cited but, in my opinion, contain confusions about the locus of propensities and their connection to long-run frequencies. The same is true of Peirce; see Suárez (2013) for extensive discussion.

3 - The Humean Mosaic (HM) is basically everything that happens, throughout all space and time. It is meant to include the objective description of all situations at all places and times, in terms of "occurrent" properties - that is, excluding purely dispositional properties, powers, necessitation relations (if any) between events, and other such things about which Humeans are notoriously skeptical. The HM is easier to imagine in line with a classical-physics worldview. It could contain point-particle trajectories, mass density distributions over space and time, charge distributions, scalar and vector fields, and so forth. It can contain things like colors and beliefs also, if we have a way of reducing them to non-dispositional properties on (parts of) the mosaic. Since primitive propensities are usually thought of as a type of disposition, they are of course not to be found in the Mosaic, according to Humeans at least; and the point of the present dialectic is precisely to see if there is a way, indirectly or directly, to understand what it would be for the HM of a world to contain chance propensities.
} 


\section{OBJECTIVE CHANCE: NOT PROPENSITY, MAYBE DETERMINISM}

ning with Hume and continuing today pursues this option. ${ }^{4}$

So most non-Humean philosophers who believe in laws of nature at all would like to ascribe them an intermediate-strength modality: physical necessity. How physical necessity should be understood is a thorny issue, which we need not explore. But it is worth noticing that philosophers and physicists at least make attempts, from time to time, to provide a deeper account of the nature and source of the physical necessity of laws. In recent analytic philosophy it has become fashionable again to argue that the necessity of physical laws is grounded in the essential natures of the basic physical kinds, and their powers or dispositions. Without an illuminating further account of these notions (essence, power, nature), of course, this is a relatively shallow account, and one that Humeans will reject. But it is something. As Filomeno (2014) discusses, there are much less shallow - though still highly speculative, and not yet successful - attempts at times by physicists to give a derivation of the necessity of the physical laws of our world. The point of mentioning such efforts is that they show that, when it comes to the modal aspect of laws, it seems at least conceivable that a substantial reductive account could be given, even if none has yet been achieved.

Whether or not any such programme of explaining or deriving the necessity of laws of nature proves workable, though, philosophers will presumably not despair about non-chancy laws of nature, and for a perfectly good reason. At least part of the content of law claims seems clear: the part that says what actually is the case. Now, what about chancy laws?

We might expect that chancy laws also have two sides to their content: actuality-related and modal. Van Fraassen (1980, ch. 6) and others certainly think of objective probability as involving a new sort of modality, intermediate between possibility and necessity, and equipped with its own logical rules, namely the probability calculus. So let's see if we can separate out the actuality-related and modal parts of the content of a probabilistic law.

Just as regular laws have a canonical logical form, “All F's are G's", probabilistic laws will have a canonical form too: $\operatorname{Pr}\left(G_{i} \mid S\right)=x_{i}$, or perhaps $\operatorname{Pr}_{S}\left(G_{i}\right)=x_{i}$. As above, the $G_{i}$ are the possible "outcomes" of the chance process covered by the law, and the law gives us their objective chances.

Let's consider first the modal content of such laws. Do we understand it? It is not supposed to be mere possibility; true, we generally take it that any of the outcomes $G_{i}$ that have non-zero chance are "possible", but that is not what we take to be the real story. The real modal part is supposed to have something to do with the number $x_{i}$, and be "stronger" the closer $x_{i}$ is to 1 . What is this modality that comes in a continuum of relative strengths? Do we capture it by saying, for example, "Since $\operatorname{Pr}\left(G_{1}\right)$ is twice as large as $\operatorname{Pr}\left(G_{2}\right)$, it is twice as possible."? No - this is meaningless. Possibility itself does not come in degrees; an event is either possible, or it is not. Probability or "likelihood" does come in degrees, but probability, in the primitive-chance sense here at issue, is precisely what we are trying to analyze or interpret.

The intended modality here is often, in a loose way, cashed out in terms of a counterfactual - a statement about what would happen. The kind of thing we want to say goes like this: "If the $S$ conditions were to be instantiated repeatedly, in a large number of causally independent "trials", then $G_{i}$ would be the outcome in approximately an $x_{i}$ fraction of the trials." We say this, but then we immediately take it back: "Of course, this need not always happen; it is simply very likely to occur, with higher and higher probability as the number of trials increases." But now our counterfactual explication of the modal content of the law is in trouble. We will require an explication of the meaning of this new probability claim - both its actuality-related part (if it has any), and its modal part - and it is easy to see that we will be mired in an endless regress. So, the modal part of a chancy law does not seem to have any clear content. We want to be able to cash it out in terms of something about what would - definitely - happen in certain circumstances, but we cannot.

\section{Digression: a way to cash out modal content?}

There is one more way to try to cash out the modal part of chancy laws' content, which has been explored in different ways by authors such as McCall (1994), Fetzer and Nute (1979), Giere (1976) and Pollock (1990). The idea is to analyze the objective chance as being some sort of proportion, among the branches representing possible futures of our world (McCall), among possible but non-actual "objects” or trials (Pollock), or among possible worlds (Giere, Fetzer). The idea seems promising at first: provide a non-empty semantic content for propensity ascriptions, one that at the same time makes clear the modal aspect of that content! Unfortunately, this rosy prospect endures only for as long as it takes to spell out one (any one) of these modal-proportion proposals.

Pollock proposed to identify $\operatorname{Pr}(A \mid S)=x$ with $<$ The proportion of physically possible $S$ 's that are $A$ 's is $x>$. We are to imagine a number of possible worlds, sharing the laws of our world, in which the relevant setup or conditions $S$ are instantiated; and the proportion of those worlds in which $A$ results is the objective probability we are trying to explicate. Based on Pollock's theory, we might say that the modal content of primitive chance statements is this: a fraction $x$ of all (relevant) possible $S$ - worlds are $A$ - worlds. McCall, by con- 


\section{OBJECTIVE CHANCE: NOT PROPENSITY, MAYBE DETERMINISM}

trast, proposes that we think of reality as having (literally?) a branching-tree structure, where the past up to now is the trunk and the branches sprouting from now towards the future represent possible ways things can go. In the branching structure, there will generally be multiple A-branches and multiple branches for the other possible outcomes of the $S$ setup; the objective chance of $A$ in $S$ is then identified with the proportion of $A$-branches out of the total number of branches starting with future chancy setup $S$. The diagram below, adapted from van Fraassen (1989, p. 84), illustrates McCall's proposal. Fetzer and Giere also propose accounts that equate, in some sense or other, primitive chances with ratios or proportions or relative weights of sets of possible worlds. ${ }^{5}$

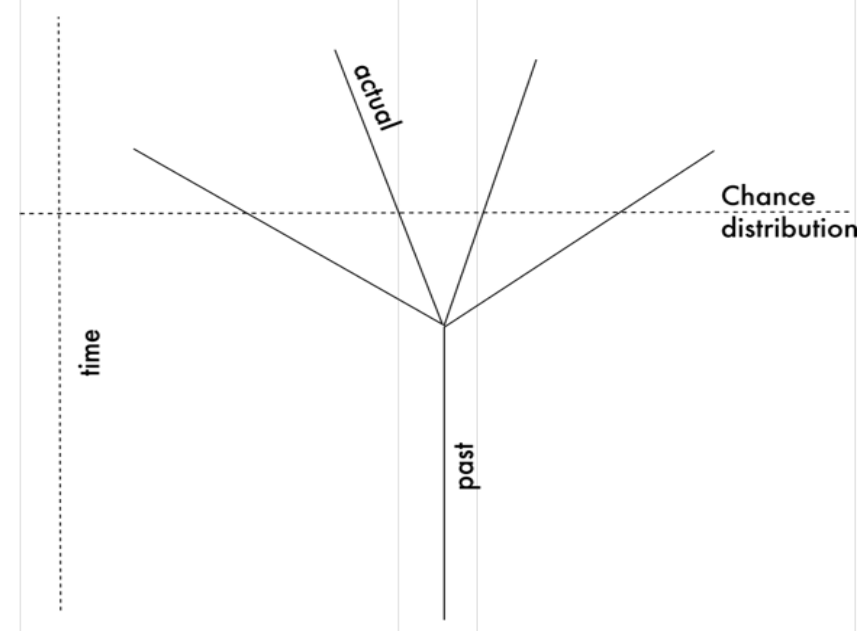

Figure 1: The horizontal-vertical problem

These explications of chance each have internal difficulties, some or all of which were discussed back in the 1970 s when most of these works were done. But in addition, as a genre they face a few difficulties that seem to me insuperable.

In order to capture chances that take on any real value (i.e., irrational numbers, as well as rationals such as $1 / 3$ ), the authors are forced to posit infinite structures (in some cases countable, in others, continua). But ratios in infinite sets are notoriously problematic, being either undefined or requiring ad hoc assumptions or additions to the structure. Giere (1976), for example, ends up using continua in his semantic constructions, and positing ad hoc weight functions over sets of possible worlds. ${ }^{6}$

But the worst problem that all these proposals run into is what van Fraassen called the horizontal-vertical problem.
What these proposals give us is a ratio when we "cut across" the relevant structure of possibilities horizontally, as in the diagram above. But the actual world is just one path through that upward branching tree, or one world in the whole set of possible worlds. In order for objective chances to guide our expectations in the way we want, we need to be sure they reflect, as appropriate, the vertical frequency of $A$ 's in $S$-situations, the frequency found in the actual world. What reason do we have to think that the horizontal ratios or measures offered by these accounts are indicative of the vertical frequencies in the real world?

This last issue touches on a key aspect of the dialectics of primitive chances that we will explore further shortly. For now, though, we just need to take away the following upshot. Various authors have attempted to cash out the meaning of propensity-type chance claims in terms of ratios among sets of possible worlds or possible objects. But the elaborate mathematical constructions they created for this task all end up not being something we can take literally and seriously as ontology, and at best they serve as structures in which one can find a way to embed and represent the numerical chances that we started out wanting to understand. They do not fulfil the task of letting us understand the modal part of the content of primitive chance claims, and even if they did, it would remain a mystery why the chances so defined should tell us anything about what will happen in the actual world. End digression

Perhaps, as we found with non-chancy laws, at least the actuality-related content of a chancy law statement is clear? Unfortunately, it is not. Or at least, nothing capturing the numerical aspect appears to be in the offing. The actuality-related part cannot be merely the claim that whenever conditions $S$ are instantiated, one of the $G_{i}$ results. ${ }^{7}$ That would not capture the numerical content of the $x_{i}$. But we equally well know we cannot take the law to be saying that, in actual cases where $S$ is instantiated, a proportion $x_{i}$ of the outcomes are $G_{i}$. An actual frequentist, (the simplest Humean), can say this, but not the propensity advocate. The actual frequencies may - in general, we expect, they will - diverge from the objective probabilities, especially when the number of actual cases in all of history is low. But if not this, then what is the actuality-related content of a chancy law? As far as I can see, there is none - nothing that is clear, true, and captures in some way the numerical-strength aspect of the chances. Ordinary laws of nature, on a straightforward realist approach, have a clear-cut actuality-related content, and a modal con-

5- Of these authors, although all four purport in some sense to be providing the meaning (or at least the 'semantics') of propensity ascriptions, once the apparati have been presented only McCall seems to offer his in a serious vein, and about even his account I am not sure how literally we are meant to take it. Giere and Pollock both characterize their formal constructions as merely heuristic devices, and assure the reader that their framework of possible worlds/objects and measures over sets of them is not to be taken literally, but merely as a tool to represent propensity facts in a mathematically useful form.

6- Giere essentially concedes the points I am making in his summary (p. 332), writing: "Metaphysically speaking, then, what are physical propensities? They are weights over physically possible final states of stochastic trials - weights that generate a probability distribution over sets of states. The function u provides only a formal and rather shallow analysis of this distribution. But is it not just the task of a propensity interpretation to explain what these weights are? No, because it cannot be done." The reader is left frankly puzzled about why an elaborate formal apparatus was needed, if in the end it is not to be taken seriously and does not shed any actual light on what it means to say that 'S has a propensity of o.x to give rise to A'.

7-In fact, even this is not necessarily so; the coin may, after all, land on its edge. 


\section{OBJECTIVE CHANCE: NOT PROPENSITY, MAYBE DETERMINISM}

tent that remains obscure. But chancy laws, on a straightforward realist approach, have no clear content of either sort. ${ }^{8}$

In order to try to find the content of chance law statements, I divided the search into two parts, one actuality-based and one modal; and we found it difficult to identify any clear content of either sort. But perhaps the problem is not with chance laws, but rather this proposed division of content itself. What happens if we go looking for the content of chance-giving laws simpliciter, without trying to split the modal from the actual/ factual? I see no new options on the horizon. It seems that if we cannot offer a translation of (part or all of) the content of a chance-law claim into something about frequencies of outcomes, we must instead stick to the notion of the probability of those outcomes. But this is just to leave un-explicated the very notion whose meaningfulness is at issue.

Now is the time to tackle head-on the likely primitivist response to the above: the whole search for a translation of the meaning of chance claims or chance laws in terms of something else was misguided. The whole point of positing objective chances or chance laws as a primitive is to make clear that it's not possible to give an explication in terms of something else. Nor is it needed; we (most of us!) understand perfectly well what is meant in making claims about objective chances, how to verify or falsify them, and how to use those claims. As Maudlin asks, "What more, exactly, could be wanted?".9 With this we enter phase 2 of the dialectics.

Let's start with the primitivism espoused by Elliott Sober, which he calls the "No-theory theory" of probability. The basic idea of the no-theory theory is that, in some cases at least, objective probability is not analyzable in other terms, and instead are a perfectly respectable theoretical concept. And like many important theoretical concepts, probability may not be definable in terms of other concepts, yet be perfectly well implicitly defined by its role in the theories that use it.

Sober (2010) offers us the analogy of the concept of (inertial) mass in physics. Despite the absence of a clean and clear definition, we believe that mass is an objective property of many physical objects, and for good reasons. For example, we have various different techniques for measuring or estimating mass, and they converge satisfactorily in most applications. The best explanation of this convergence is that it is due to a common cause, namely, that there is an objective property of mass (or rather: a certain quantity of mass) in the objects being measured. So even though we might consider that inertial mass is an unobservable property, perhaps even an intrinsically dispositional property, we can have perfectly respectable grounds for believing in its existence.

I find Sober's use of the theoretical concept of mass as an analogy for propensity-type probability unpersuasive. Mass is a property that, for a wide range of values, can be directly (if roughly) judged by human senses, and accurately measured with easily portable devices (e.g. scales). If a wide class of objective probabilities could be rapidly and accurately estimated by simple devices that we (say) insert the chance setups into ${ }^{10}$, then there would no doubt be a widespread and generalized sentiment among philosophers and scientists that the lack of an explicit theory was no serious obstacle to believing in the objective reality of probabilities.

But, of course, the real situation is quite different. There are no such devices. Moreover, there is no way to mount the sort of common-cause argument that Sober invokes as grounds for believing in the unobservable quantitative property of mass. When it comes to chances, we have exactly one way of measuring them: by looking at frequencies in (what we hope are) repeated instances of identical setups. ${ }^{11}$ Everybody believes in mass and in its objectivity, even if a few peripheral applications are disputable. But among philosophers of probability, and scientists and statisticians who use it, there are substantial numbers who don't believe the concept picks out any objective reality at all (over and above actual frequencies). This sociological difference reflects the very real disanalogies between the concept of mass and the concept of primitive chance.

But if Sober's analogy is not quite convincing on its own, we still have to face the fact that many primitivists feel that they perfectly well understand the meaning of claims like $\operatorname{Pr}\left(G_{i} \mid S\right)$ $=x_{i}$, ' and add that we understand both how to use them and how to confirm or disconfirm them. How could that be so, if my claim that we don't understand primitive chance claims were correct?

The key to this part of the dialectics is the Principal Principle: the primitivists are relying on it, implicitly or explicitly, but the skeptic says they have no right to do so.

We can express one version of the PP in symbols, as follows.

Let $\mathrm{Cr}\left(\mathrm{L}_{\_}\right)$be a rational subjective probability function (credence function), $A$ be a proposition in the domain of

8 - The qualification "on a straightforward realist approach" here is meant to exclude approaches to laws that demote them to something essentially to do with humans, e.g. instrumentalism, "inference-ticket" accounts, etc; and also to exclude Humean reductive accounts of laws such as that in Lewis (1994). 9 - Maudlin (2007), "What could be objective about probabilities?".

10 - There is a class of objective chance setups for which we seem able to judge the chances rapidly and accurately: classical gambling devices for which we feel we can deduce the chances on the basis of symmetry considerations. But this is a matter of inferring, not measuring; and as I will argue later, such systems are probably best understood as possessing objective probabilities grounded in determinism plus initial-condition facts, not primitive propensities.

11 - That said, with the advent of QM we have a new technique for coming to know certain objective chances: namely, deriving them from quantum wave functions, cross-sections and so forth. (Something similar is true for probabilities given by classical statistical mechanics, but for most philosophers those are not considered candidates for being fundamental or primitive chances.) This novelty strengthens the grounds for considering QM chances to be objective and mind-independent, but does not at all resolve the puzzle about what propensity-type chance claims could mean. 


\section{OBJECTIVE CHANCE: NOT PROPENSITY, MAYBE DETERMINISM}

an objective chance function $P\left(\_\right.$), $E$ be the rest of the agent's background knowledge, assumed to be "admissible" with respect to $A$, and $X$ be the proposition stating that $P(A)=x$. Then:

$$
\text { (PP) } \operatorname{Cr}(A \mid X E)=x
$$

If a rational agent believes herself to know the objective probability of $A, P(A)=x$, then that will be her subjective degree of belief in $A$, conditional on that fact. If $x$ is near 1 , the agent confidently expects $A$ to be true; if $x$ is low, the agent confidently plans on $A$ not coming out true; and so forth.

Plugging primitive chance probabilities into PP gives us the feeling that we understand the actuality-related content of the chance law: it is telling us that we should have such-andso degrees of belief, so-and-such expectations for sets of repeated trials, etc. The PP is also used, implicitly, when we go from facts about frequencies in repeated trials to beliefs about what the objective chances are. We become $95 \%$ confident, e.g., that the objective chance is within 0.1 of $x$ after seeing a frequency of $x$ in a certain number of trials. This subjective probability is justified (if it is justified!) by the fact that it comes out of application of PP. So it seems that the primitivists are right after all: PP supplies the missing content to primitive chance statements, and its tacit invocation explains why most philosophers have felt it obvious that such statements do indeed make clear and contentful claims about the world. But as the quote from van Fraassen at the start of this section indicated, to say how and why beliefs about primitive chances should shape our expectations about what will happen appears to be impossible.

Here we arrive at a crucial step in the dialectics of primitive chance, and there are two points that I want to make.

First, from the response on behalf of primitivism we are now considering, it would seem that the appeal to PP is carrying the entire weight of providing content to primitive chance claims. That means that we are being asked to entertain that the whole meaning of such statements is carried by their (alleged) claim to rationally constrain our subjective credences. In other words, the content of such statements amounts to some facts about how rational agents should constrain their degrees of belief - i.e., normative facts. But this means that the content seems to fall on the wrong side of the fact/value divide.

More to the point, and this is the second and decisive response, we have just traded one mystery for a slightly-different one. If it is a fact that rational agents should conform their credences to the values of the primitive chances - if they can come to know the latter, at least - then this ought to be true because of some feature or property that the primitive chances possess! And the connection cannot just be blindly asserted; it needs to be established by argument or demonstration.

Now is the time to come back to the famous quote from Lewis mentioned earlier. Referring to the possibility of positing intrinsically probabilistic dispositions, i.e. chance propensities, Lewis writes:

Be my guest - posit all the primitive unHumean whatnots you like. (I only ask that your alleged truths should supervene on being). But play fair in naming your whatnots. Don't call any alleged feature of reality 'chance' unless you've already shown that you have something, knowledge of which could constrain rational credence. (1994, p. 484).

The problem is that there is no way for the advocate of primitive chances to meet this challenge. Their alleged primitive chances, precisely by being bare primitives, lack any content beyond their numerical values. And being told that a system $S$ has a whatnot-property $G$ such that $S$ 's future possible behavior $O$ has a numerical $G$-value $x$ tells us nothing whatsoever that can or should clearly constrain our rational credences concerning $O$ 's occurrence or non-occurrence. ${ }^{12}$

To some philosophers it seems clear that the challenge here laid down to the advocate of primitive chances is unfair. Hall responds to Lewis's challenge thus:

What I 'have' are objective chances, which, I assert, are simply an extra ingredient of metaphysical reality, not to be 'reduced' to anything else. What's more, it is just a basic conceptual truth about chance that it is the sort of thing, knowledge of which constrains rational credence. Of course, it is a further question - one that certainly cannot be answered definitively from the armchair - whether nature provides anything that answers to our concept of chance. But we have long since learned to live with such modest sceptical worries. And at any rate, they are irrelevant to the question at issue, which is whether my primitivist position can 'rationalize' the Principal Principle. The answer, manifestly, is that it can: for, again, it is part of my position that this principle states an analytic truth about chance and rational credence. (2004, p. 106).

Hall's response to the challenge has, it seems to me, all the virtues of theft over honest toil. This form of argument is not one that we should be happy to extend to other areas of philosophical debate. Consider a debate between philosophers who posit a conscious mind, but one side considers it clear that conscious minds are non-physical, while the other doubts this and asks for a demonstration. "It is just a basic conceptual truth about conscious minds that they are non-physical 


\section{OBJECTIVE CHANCE: NOT PROPENSITY, MAYBE DETERMINISM}

things. Of course, it is a further question - one that certainly cannot be answered definitively from the armchair - whether nature actually contains conscious minds. But we have long since learned to live with such modest sceptical worries." The opponent will regard this move as a cheat, and feel that while we have plenty of evidence that something deserving the epithet 'conscious mind' exists, it is far from obvious that it is a non-physical thing (whatever that might mean, something about which the opponent will no doubt ask for clarification). Mutatis mutandis, this is the perspective I maintain concerning objective chance.

There is one more turn of the dialectical wheel to consider before we move on. While Hall's move may cross the lines of fair play, as Lewis thought, there is a somewhat more modest way of postulating objective chances that does not. In his (1995) The Facts of Causation, Hugh Mellor postulates what he takes to be something like the propensity theorist's objective chances, but he explicitly defines such chances as that feature of reality, whatever it may be, that fits our conceptual notion of chance, especially in the PP role of being such as to rationally constrain credence. Mellor's move differs from Hall's in a crucial respect: it leaves open the possibility that something other than a primitive feature of reality - something, that is, that might satisfy the reductivist desires of Lewis - could turn out to be what "objective chance" actually refers to.

What Lewis (1994) tried to show is that this is in fact the case: a reductive story concerning the notion of objective chance can be told, along Humean Best System lines, which succeeds in capturing most of what we ever wanted the notion of chance to do for us. Hoefer (2007) and Frigg and Hoefer (2015) develop a pragmatic version of such a Humean theory, and show explicitly that 'objective chance' as so defined should constrain rational credence in just the way captured by PP.

Rather than explaining my or Lewis' Humean approach in detail, what I want to do in the rest of this paper is give further motivation for rejecting the primitive chance approach by showing how easily objective probabilities can arise in the context of underlying fully deterministic laws. It is an open possibility, as we will see, that perhaps all objective chances can be seen as arising in this way.

\section{4 - Chances from Determin- ism: the Solution?}

Why do some philosophers postulate the existence of primitive, irreducible "extra ingredients" of reality, over and above the non-controversial physical things like tables and chairs and human bodies? In most cases, they are making a move of inference to the best explanation (IBE). In the case of propensities or chance laws, the explanandum is supposed to be the existence in our world of stable probabilistic regularities associated with certain kinds of physical situations or setups. By 'stable probabilistic regularities' what I mean is: patterns of frequencies, in medium-to-large collections of instantiations of the relevant setup kind, of the type that one expects (via $\mathrm{PP}$ ) given certain objective probabilities.

In a recent paper on chance and determinism, Nina Emery (2015) discusses this sort of claim, that chances (including, presumably, primitive chances) may be posited in order to explain facts about event frequencies. Her discussion centers on a "paradigm case" of objective chance, the up-deflection probability of silver atoms passed through a Stern-Gerlach device. The silver atoms in Emery's paradigm case have previously been prepared in a deflected-up state along axis $x$ by previous measurement interaction, and subsequently are measured along axis $x^{\prime}$ which is not far from $x$, so the quantum probability of upward deflection is high, and indeed most atoms are deflected up. Emery stipulates that no hidden variable differentiates the prepared silver atoms in a way that could explain specific outcomes; this stipulation is part of what makes it her "paradigm case". Turning to explanation, Emery writes:

[There is a] more general claim which, given the observations she has made, is liable to be part of Sally's best theory about the experimental set-up:

(5) The chance of any silver atom in the experiment being deflected up is very high.

And (5) demonstrates that in addition to claims about frequency providing evidence for claims about chance, claims about chance can also, at least sometimes, explain claims about frequencies. In particular, (5) explains (6):

(6) Most of the silver atoms in the experiment have been deflected up.

That (5) explains (6) may not [be/sic] immediately obvious, but the argument for it is straightforward. First, notice that if (5) does not explain (6), then nothing does. It is part of the paradigm case that there is no feature such that all and only silver atoms that are deflected up have that feature before they are sent through the magnets. What else, then, could explain the fact that most of the silver atoms sent through the experiment are deflected up? What other sort of fact could be provided as answer to the question, 'Why have most of the silver atoms in the experiment been deflected up?' Second, notice that if nothing explains (6), then we have no reason for expecting the pattern described in (6) to continue. If it is just a massive coincidence that most of the silver atoms sent through the experiment have been deflected up, then we should not expect the next silver atom to be deflected up. But, we do expect the next silver atom to be deflected up, and most of the silver atoms after that. So we expect the pattern described in (6) to continue. So something explains (6). And the only possible explanation is (5). 


\section{OBJeCtive CHANCE: NOT PROPENSITY, MAYBE DETERMINISM}

There are a number of ways to resist Emery's argument and her conclusion. Some empiricists (though not I) would dispute her claim that if we believe nothing explains the existence of a certain regularity, then we have no reason to expect it to persist. Moreover, it is controversial whether primitive chances do provide an explanation of stable probabilistic regularities. Emery's explanans and explanandum are worded fairly vaguely, presumably deliberately, so as to accommodate the potential gap between the chance and the observed actual frequency. But the wiggle room between primitive chances and frequencies, which in principle has no bounds, can be as much foe as friend to the explanation-based defense of primitive chance. For example, suppose that the actual frequency behind Emery's (6) is 97\%, but the quantum chance is supposed to be only $89 \%$. If that frequency is built up over many thousands of silver atoms, then standard statistical practice would be to regard this frequency as strongly disconfirming, if not outright refuting, the hypothesis that the objective chance is in fact $89 \%$. As the frequency gets further and further away from the putative objective chance, intuitively, the alleged explanation of the frequency by chance gets worse and worse, eventually becoming no explanation at all; but the (null) semantic content of a primitive chance claim gives us no clue about how to justify or quantify this intuitive claim. This brings us back to the considerations of the first sections of this paper, which can be read as an extended argument that primitive chance claims cannot explain actual frequencies or our expectations about them, because (i) we don't know what the semantic content of primitive chance claims could be, and (ii) we have no justification for plugging them into PP to generate subjective expectations.

But setting aside all these objections, what I want to address now is the following question. Even if primitive chances provide $a$ possible explanation for stable probabilistic regularities, are they in fact the best explanation? I will argue that they are not: deterministic underpinnings do the explanatory job at least as well, or better.

There are at least two different routes for extracting objective probabilities from underlying deterministic mechanics. But at a certain level of description the basic idea for both routes is the same: if the dynamics of a system is such that small differences in initial conditions lead to very big differences in the "output" state, then an enormously wide variety of distributions of such initial conditions (over the relevant space of possible initial conditions for the type of system) will all lead to approximately the same stable probabilistic regularities in the outputs of the system. ${ }^{13}$ istic coin-flip situation, as modeled in (Diaconis, Holmes and Montgomery 2007). In their mathematical coin flip model, which they also implement experimentally, whether a coin initially placed heads-up into the coin flipping mechanism will land heads depends deterministically on two variables, the initial upward velocity and the initial rate of rotation (spinning), as illustrated in their diagram below. If the initial conditions (IC's) fall into a shaded area, the coin lands heads; if they fall in a white area, it lands tails. Note that the area of the shaded regions, in the magnified region of IC space, is approximately the same as the area of the white regions:

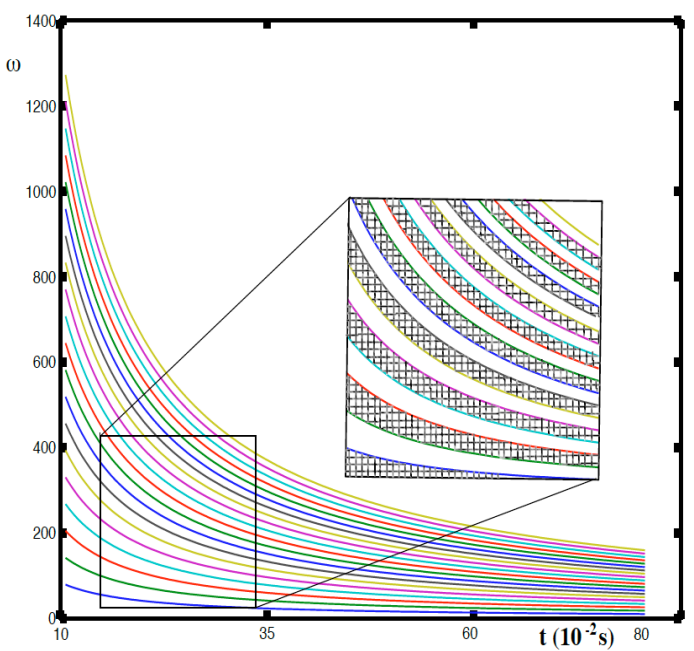

Figure 2: Hyperbolas as defined by the various initial values of $\omega$.

Now imagine that the initial conditions of coin flips all fall in a blob-shaped sub-area of the square region magnified in their diagram. Suppose further - as is actually the case, presumably, in real coin flips - that it is difficult to control exactly where the initial condition of a flip lies, in this region, and hence that the pattern of actual initial conditions, when real people flip coins (say) 100 times, looks like 100 points scattered randomly over that blob-shaped sub-area. Being so scattered in a random-looking way, uncorrelated with the bands of shaded or un-shaded regions, about half of the flip IC's will fall in a shaded region, and half in a white region. This yields, and explains, the $50 \%$ chance of heads we commonly ascribe to coin flips. ${ }^{14}$

This basic idea is well illustrated by a very simple, determin-

13 - This basic idea goes back at least to work by Poincaré (1896/1912) and has been developed and extended to many concrete systems in subsequent decades. The "wide variety of distributions" on initial conditions mentioned above, which all yield essentially the same macroscopic statistical behavior, is reflected in Poincarés name for the phenomenon: the "method of arbitrary functions". (Though it is not strictly true that the normal statistical behavior emerges for arbitrary functions.)

14- Why should the distribution of IC's over the blob-shaped area be random-looking and not correlated with the hyperbolic-shaped bands? Aside from the fact already mentioned, that it is hard for us to control the IC precisely, there need be no answer to this; it just so happens that real coin flips do display this lack of correlation. (And the same holds for the IC's of many other deterministic systems that amplify small IC-differences into large macro-outcome differences.) 


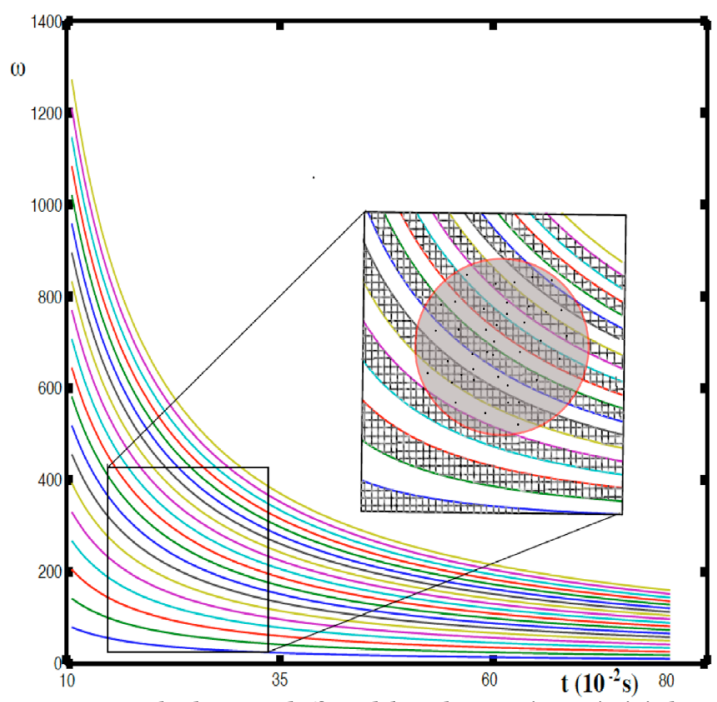

Figure 3: Hyperbolas as defined by the various initial values of $\omega$.

In the second diagram, we depict a blob in which all the actual coin flip IC's fall. We may wonder: if we approximate the distribution of such points over that region of IC-space with a continuous probability density, what will that function look like? Will it be a uniform distribution, or peaked near the center, or some other distribution entirely? Given the approximately equal areas of white and shaded bands inside the blob, a uniform distribution guarantees the expected 50/50 probability outcome. ${ }^{15}$ But many other distributions will do the same. One would only expect a distribution to diverge significantly in its results if it were systematically correlated with the hyperbola-shaped bands, e.g., higher in value over dark bands and lower over the white bands, or very sharply peaked around a certain specific IC value in the middle of one of the bands.

The coin flip case illustrates the basic idea, of which we will now briefly look at two refinements. 1. Stochastic nomological machines. The Humean account of chance that I favor considers all objective probabilities to be grounded, ultimately, in facts about the Humean mosaic. Particularly important are facts about the patterns and frequencies of various types of events, of course. And if we think about the features of the Humean mosaic at a microscopic level, one very important fact stands out: for oft-repeated types of situations or setups which involve outcomes determined partly or wholly by the (relatively) microscopic initial conditions of the components of the setup, it seems to be the case that the patterns of the actual values of those IC's are usually "noisy" or random-looking, while approximat-

\section{OBJECTIVE CHANCE: NOT PROPENSITY, MAYBE DETERMINISM}

ing some overall smoothly-varying distribution. (One example of this is the ubiquitous Gaussian or bell-curve distribution, characteristic of so many micro- and macro-level phenomena.) In (Hoefer 2007) I named this apparent fact about our world the 'Stochasticity Postulate' (SP). ${ }^{16}$ For many types of system, the way that the IC's determine or partly-determine the outcome of the chance setup entails that (random-looking IC's IN $\rightarrow$ random-looking outcomes OUT), i.e. stable probabilistic regularity, just as we saw for the coin flip case. Adapting Cartwright (1999)'s notion of a 'nomological machine', I call these sorts of systems 'stochastic nomological machines' (SNM's). All the usual classical gambling devices can be considered stochastic nomological machines, and perhaps also many other phenomena where we are inclined to attribute stable objective probabilities. The ground of the objective chances is not a primitively chancy disposition, but rather a ubiquitous fact in our HM about how IC's tend to be distributed in random-looking ways, and results determined deterministically or quasi-deterministically from those IC's. Strevens $(1998,2003)$ provides an extended treatment of this way in which chance can be grounded in determinism.

2. Epistemic chances (Myrvold). In some recent works, Wayne Myrvold (see 2012, 2016) proposes to ground the objective probabilities of Statistical Mechanics, as well as the classical gambling-type systems such as coin flips or wheels of fortune, on the same basic facts we have seen just above. The difference in Myrvold's approach is that he does not directly ground the objectivity of his chances on what I called the Stochasticity Postulate, but rather on facts about how SNM's are structurally such as to force rational agents' subjective degrees of belief (or 'credences') about outcomes into agreement. ${ }^{17}$

The idea is that, for certain systems, a wide range of probability distributions [over IC's] will be taken, via the dynamics of the system, into distributions that yield approximately the same probabilities for some statements about the system. Poincaré called the input probability distributions "conventions," which leaves their status unclear. In this paper, we take up a suggestion of Savage (1973), that these be regarded as subjective probabilities. The idea is that the evolution of the system can wash out considerable differences in credences about the initial state, if we restrict ourselves, not to all propositions about the system at a later time, but a limited class of them. Typically the propositions of interest will be those whose truth-values can be ascertained via feasible measurements. [...] If it so happens that the dynamics of the situation wash out differences between credence-functions of any two reason-

15 - Actually not quite 50\%/50\%. Diaconis et al. op cit. show that there is a slight bias of near $1 \%$ in favor of heads, if the coins start with heads facing up; hence the title of their article.

16-I chose the potentially misleading term 'postulate' because it is meant to be a fact about IC's all over the world at all times, and we only have direct evidence for it being the case around here (cosmically speaking) and in our past light cone.

17 - Myrvold prefers to reserve the term 'objective chance' for the kind of probability, if any, that involves intrinsic and fundamental indeterminism; in other words, primitive propensities or chancy laws. In addition to 'epistemic chances', Myrvold also uses the term 'almost objective probabilities' for the determinism-based probabilities we are discussing here. 


\section{OBJeCtive CHANCE: NOT PROPENSITY, MAYBE DETERMINISM}

able agents, with regards to the results of feasible experiments, we cannot come to this conclusion on the basis of either epistemic considerations or physical considerations alone. On the epistemic side, we require a restriction to a class of probability-functions that can represent the belief-states of reasonable agents with limited access to information about the system, and we require a limitation to certain sorts of measurements, since, for an isolated system, no invertible dynamics will wash out all differences between probability functions. On the physical side, we need the right sort of dynamics. If it is the case that the dynamics lead to probability distributions that yield approximately the same probabilities for the results of certain measurements, given as input any reasonable credence-function, then the values of these probabilities will be a matter of physics. We will call the probabilities that emerge in such a scenario almost objective probabilities, or epistemic chances. (2012, pp. 2-3).

An important question about the idea of grounding objective chances on underlying determinism is this: what about the chances of quantum physics? They are surely among the best, if not the best, candidates for being truly objective chances in the world. But conventional wisdom has it that quantum physics is intrinsically and fundamentally indeterministic. Is there a way to base quantum chances on underlying determinism?

The answer is: it's too early to tell. Nowadays most physicists and philosophers of physics have become aware of the alternative version of non-relativistic QM known as "Bohmian mechanics" (BM), discovered by David Bohm in 1952. In that theory all particles have definite, precise positions at all times, their trajectories over time being determined fully by the quantum state (or "wave function"), which evolves deterministically according to the Schrödinger equation, and the equally deterministic "guidance equation" postulated by Bohm. The details of Bohmian mechanics are beyond our scope here, but it is noteworthy that it makes all the same predictions as standard QM, if we add one further postulate: the "quantum equilibrium hypothesis", which basically says that the initial positions of all particles are spatially distributed in proportion to the size of the square of the wave function. It can be proven that once this condition is in place, future evolution of the particle positions will maintain the condition; and that, given this condition, the predictions of Bohmian mechanics are empirically indistinguishable from those of standard QM. ${ }^{18}$

Using Bohmian Mechanics, even the most striking examples of apparently primitive propensity-type chances can be brought back under the sway of determinism. This is so for radioactive decay (cited by Suárez (2013) in his defense of a propensity-based view of chance), and for the spin measurements example of Emery's "paradigm case". ${ }^{19}$ The illustration below, borrowed from (2014), shows how Bohmian me- chanics can explain a regularity like Emery's (6). Particles with initial vertical position above a certain line will get deflected upward, those with positions below that line will go downward; and as long as the particle initial positions are distributed randomly but in rough concordance with the weights given by $\left|P s i^{2}\right|$, the frequencies of upward deflections will be in good accord with the quantum probabilities.

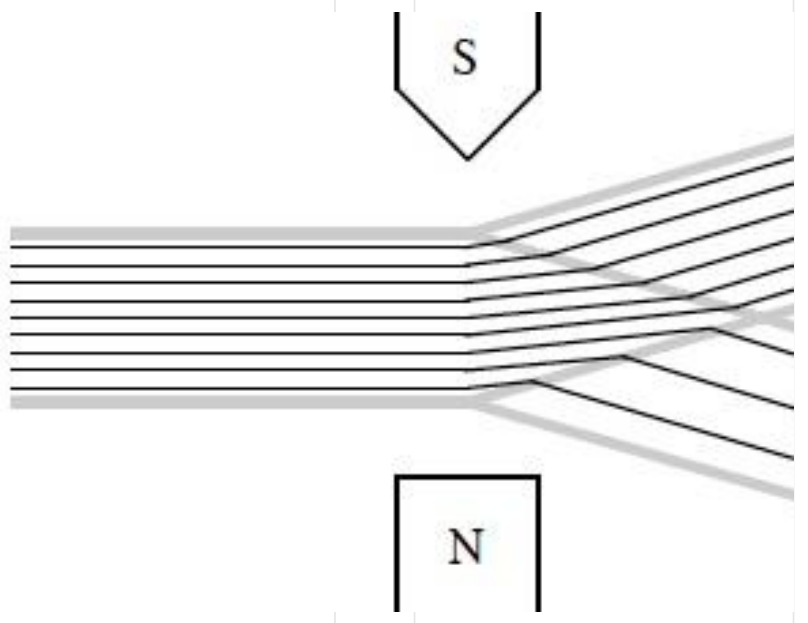

Figure 4: Initial positions of Bohmian silver atoms determine subsequent trajectory, from (Norsen 2013).

The quantum equilibrium hypothesis can be thought of as an allied condition complementing the Stochasticity Postulate in a Humean understanding of quantum chances. In this way, the Humean approach fits nicely with Bohmian mechanics. It is not clear whether the same is true for Myrvold's epistemic chances. In order for the probabilities given in BM to be epistemic chances in Myrvold's sense, it would have to be the case that starting from a huge variety of subjective probability distributions over the initial conditions for particle positions, the dynamics of $\mathrm{BM}$ forces those distributions quickly to be nearly identical to those of the quantum equilibrium hypothesis. To my knowledge, no general result of this kind has yet been proven.

Conceptual objections can be raised against BM, however (as is true of all versions and interpretations of QM), and more importantly, there is at present no widely endorsed, deterministic BM-like alternative to quantum field theories such as QED and QCD. So it is unclear whether quantum chances can be entirely grounded on underlying determinism.

But let's suppose for a moment that the Bohmian program proves extendible to cover quantum field theories, and thus that we can imagine that perhaps all chancy-looking quantum phenomena turn out to be explicable on the basis of a deterministic Bohmian physical theory plus ap- 


\section{OBJECTIVE CHANCE: NOT PROPENSITY, MAYBE DETERMINISM}

propriate "random-looking initial conditions" assumptions. Would we then have simply another possible explanation of such phenomena, a rival to stand alongside the postulation of primitive propensities or chancy quantum laws? Or would we rather have a better explanation, and hence the apparent winner in the game of IBE?

I believe the latter is the correct answer, given the problems of chance primitivism discussed in the first parts of this paper. But against this claim, the following objection may be raised: the determinist explanation here is really no explanation at all, because it just pushes the mystery over into the facts about initial conditions. Why are initial conditions in our world so reliably random-looking, as the Stochasticity Postulate asserts? Don't we now need an explanation of that fact; and might we need to invoke primitive chanciness to get that explanation, after all? ${ }^{20}$

I have no definitive response to this objection, but there are a few things that can be said. First of all, it is an accepted truism in philosophy of science that demands for explanation can always be raised at a new level, once an explanation has been offered at one level. Newton's theory of gravity explained the orbits of the planets; but one can still ask why it is a law that all massive bodies attract each other in proportion to their masses and in inverse proportion to the square of the distance between them, rather than according to some different mathematical formula. Explanation always runs out at some point, but usually this does not make us feel that the extra level or levels of explanation that science often gives us have no value. Second, although stopping once we reach a fundamental law may feel less unsatisfactory than stopping at a brute fact about initial conditions, a number of philosophers would argue that we should try to shake off that feeling. Initial conditions, especially those of the universe as a whole, are both explanatorily unavoidable posits, and not appropriate targets for further explanatory demand. (See (Callender 2004) for a defense of this perspective.) And finally, we can note that the general character of the initial conditions posited by my Stochasticity Postulate involves nothing particularly special or contrived; quite the opposite, they are conditions that can be described as 'typical', in a mathematically precise sense. There are philosophers working on both classical Statistical Mechanics and Bohmian Mechanics who try to use considerations of the typicality of initial conditions that give rise to the normal/expected behavior to argue that these sciences do offer impeccable explanations of such behavior. ${ }^{21}$ I do not want to endorse this line myself, but it is something to be kept in mind when considering whether it is a defect of deterministic explanations of chancy-looking physical systems that they have to make an assumption about initial conditions.

\section{5 - Conclusion}

In this article I have tried to induce the reader to see that it is deeply puzzling what we could be doing when we postulate primitive propensity-type chances or fundamental/irreducible chancy laws of nature. I presented what I think of as the dialectics of primitive chance, showing that every natural attempt to explain what the meaning of primitive chance-ascribing statements could be fails. Many of us feel strongly that we do understand the meanings of such statements, but it turns out that that feeling relies on taking primitive chances to be apt for guiding belief and action via the Principal Principle; and there is no way for the primitivist to justify plugging her primitive chances into PP. Primitive chances, then, cannot be posited by an inference to the best explanation argument, because their status as explainers of chancy phenomena is entirely obscure.

But even if the problems raised in the first parts of the paper are dismissed, I pointed out that one can well contest whether primitive chances give us the best explanation of the objective probabilities we usually ascribe to the world, because deriving those probabilities from underlying determinism provides a straightforward and clear explanation, without having to admit any obscure new primitives into our scientific image of the world. This is so, at least, for many classical gambling device probabilities, for Statistical Mechanics probabilities (see (Frigg and Hoefer 2015)), and for the probabilities of non-relativistic QM via Bohmian Mechanics. Whether the program of basing all physical objective chances on underlying determinism can be extended to all quantum phenomena is still uncertain. But it is a possibility that holds out hope for those of us, like myself, who have real problems understanding the alternative of primitive propensity-type chances.

\section{Acknowledgements}

I would like to thank Francesca Merlin and Nina Emery for helpful comments on draft versions of this paper. I would also like to thank members of the audience at the 2014 SPS conference in Lille for stimulating and challenging questions and discussion at my talk there, on which this paper is based. Parts of this paper are taken, with substantial modifications, from two of my earlier works on objective probability (Hoefer 2011a, 2011b).

The research work leading to this paper has been generously supported by the following Spanish MICINN \& MINECO research grants: CSD2009-0056, FFI2011-29834-Co3-03, FFI2012-37354. In 2015-16 my work was also supported by the Generalitat de Catalunya consolidated research group LOGOS, SGR 2014-SGR-81. 
REVUE

DE LA SOCIÉTÉ

DE PHILOSOPHIE

DES SCIENCES

REFERENCES

CALLENDER, Craig. 2004. Measures, Explanations and the Past: Should 'Special' Initial Conditions Be Explained? The British Journal for the Philosophy of Science, 55(2), 195-217. Article.

CARTWRIGHT, Nancy. 1999. The Dappled World. Cambridge: Cambridge University Press. Livre.

DIACONIS, Percy, HOLMES, Susan and MONTGOMERY, Richard. 2007. Dynamical Bias in the Coin Toss. SIAM Review, 49(2), 211-235. Article.

DÜRR, D., GOLDSTEIN, S. and ZHANGI, N. 1992. Quantum Equilibrium and the Origin of Absolute Uncertainty. Journal of Statistical Physics, 67, 843-907. Article.

EMERY, Nina. 2015. Chance, Possibility and Explanation. The British Journal for the Philosophy of Science, 66 (1), 95-120. Article.

FETZER, James H. \& NUTE, Donald E. 1979. Syntax, Semantics and Ontology: a Probabilistic Causal Calculus. Synthese, 40, 453495. Article.

FILOMENO, Aldo. 2014. On the possibility of stable regularities without fundamental laws. Doctoral dissertation, Autonomous University of Barcelona.

FRIGG, Roman and WERNDL, Charlotte. 2012. Demystifying Typicality. Philosophy of Science, 79 (5), 917-929. Article.

FRIGG, Roman and HOEFER, Carl. 2015. The Best Humean System for Statistical Mechanics. Erkenntnis, 80 (3 Supplement), 551574. Special issue edited by J. Berkovitz \& P. Huneman. Article. GIERE, Ronald. 1973. Objective Single-Case Probabilities and the Foundations of Statistics. In SUPPES, P. et al., (eds.), Logic, Methodology and Philosophy of Science IV. New York: North-Holland, 467-483. Chapitre.

GIERE, Ronald. 1976. A Laplacean Formal Semantics for Single-Case Propensities. Journal of Philosophical Logic, 5, 321-353.

HÁJEK, Alan. 2012. Interpretations of Probability. The Stanford Encyclopedia of Philosophy (Winter 2012 Edition), Edward N. Zalta (ed.), URL = http://plato.stanford.edu/archives/win2012/ entries/probability-interpret/

HALL, Ned. (2004). Two Mistakes About Credence and Chance. Australasian Journal of Philosophy, 82(1), 93-111. Article. HOEFER, Carl. 2007. The Third Way on Objective Probability: A Skeptic's Guide to Objective Chance. Mind, 116, 549-96. Article. HOEFER, Carl. 2011a. Time and Chance Propensities. In Craig Cal-

\section{HISTORIQUE}

Ce texte est la version modifiée de la conférence prononcée le 25juin 2014 au Ve Congrès de la Société de philosophie des sciences ( « Métaphysique des sciences », Lille, 25-27 juin 2014).

\section{SITE WEB DE LA REVUE}

sites.uclouvain.be/latosensu/index.php/latosensu/index

ISSN 2295-8029

DOI http://dx.doi.org/10.20416/lsrsps.v3i1.603

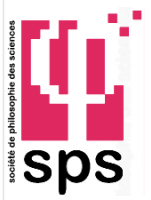

SOCIÉTÉ DE PHILOSOPHIE DES SCIENCES (SPS) École normale supérieure

45, rue d'Ulm

75005 Paris

www.sps-philoscience.org

\section{OBJECTIVE CHANCE: NOT PROPENSITY, MAYBE DETERMINISM}

lender (ed.), The Oxford Handbook of Philosophy of Time. Oxford: Oxford University Press, 68-90. Chapitre.

HOEFER, Carl. 2011b. Physics and the Humean Approach to Probability. In BEISBART, C. and HARTMANN, S. (eds.). Probabilities in Physics. Oxford: Oxford University Press, 321-337. Chapitre. LEWIS, David. 1994. Humean Supervenience Debugged. Mind, 113, 473-490. Article.

MAUDLIN, Tim. 2007. What Could Be Objective About Probabilities? Studies in History and Philosophy of Modern Physics, 38(2), 275-291. Article.

MCCALL, S. 1994. A Model of the Universe: Space-Time, Probability, and Decision. Oxford: Clarendon Press. Livre.

MELLOR, Hugh. 1995. The Facts of Causation. London: Routledge. Livre.

MYRVOLD, Wayne. 2012. Probabilities in Statistical Mechanics: What Are They? Unpublished ms. Available at http://philsci-archive.pitt.edu/9236/

MYRVOLD, Wayne. 2016. Probabilities in Statistical Mechanics. In HITCHCOCK, C and HÁJEK, A. (eds). Oxford Handbook of Probability and Philosophy. Oxford University Press. Preprint available at http://philsci-archive.pitt.edu/9957/

NORSEN, Travis. 2014. The Pilot-Wave Perspective on Spin. American Journal of Physics, 82(4), 337-348. Article.

POINCARÉ, Henrí. 1896/1912. Le calcul des probabilités. 2nd ed. 1912. Paris: Gauthier-Villars.

POLLOCK, John. 1990. Nomic Probability and the Foundations of Induction. Oxford: Oxford University Press.

SOBER, Elliott. 2010. Evolutionary Theory and the Reality of Macro Probabilities. In EELLS, Ellery and FETZER, James H. (eds.). The Place of Probability in Science. Netherlands: Springer, 133161. Chapitre.

STREVENS, Michael. 1998. Inferring probabilities from symmetries. Noûs, 32(2), 231-246. Article.

STREVENS, Michael. 2003. Bigger than Chaos: Understanding Complexity through Probability. Cambridge, MA: Harvard University Press.

SUÁREZ, Mauricio. 2013. Propensities and Pragmatism. The Journal of Philosophy, 110(2), 61 - 92. Article.

VAN FRAASSEN, Bas. 1980. The Scientific Image. Oxford: Oxford University Press. Livre.

VAN FRAASSEN, Bas. 1989. Laws and Symmetry. Oxford: Oxford University Press. Livre.

\section{CONTACT ET COORDONNÉES}

Carl Hoefer

Department of Philosophy,

University of Barcelona,

Carrer Montalegre 6,

o8001 Barcelona, Spain.

ICREA,

Pg. Lluís Companys 23,

o8010 Barcelona, Spain.

carl.hoefer@ub.edu

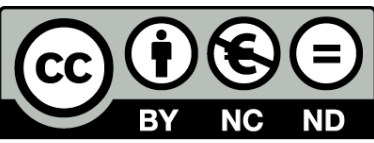

